\title{
Évaluation et contrôle des publications à l'heure du numérique et des réseaux sociaux
}

Michèle Leduc (leduc@kb.ens.fr)

Directrice de recherches émérite au CNRS, membre du comité d'éthique du CNRS

Laboratoire Kastler Brossel, École normale supérieure, 24 rue Lhomond, 75231 Paris Cedex 05

La diffusion des travaux

scientifiques subit une

profonde évolution liée

à l'usage du numérique

et à la « science ouverte »

(Open Science). Les résultats peuvent être communiqués

immédiatement et gratuitement

par dépôt sur des archives

ouvertes ou des revues en

libre accès.

Par ailleurs, la qualification

des publications par les pairs,

doublée par des critiques

constructives (le peer review)

est un système aujourd'hui

en difficulté. Ceci explique

que certains sites interactifs

aient choisi d'ouvrir un espace

de dialogue entre

les scientifiques. L'anonymat

y facilite la révélation

des pratiques douteuses dans

les publications. Des modèles

alternatifs de qualification

des publications sans éditeurs

privés se font jour.
Tout chercheur sait que la communication de ses résultats à la communauté scientifique est une étape fondamentale dans son travail. Depuis le $17^{\mathrm{e}}$ siècle et jusqu'à il y a environ une quinzaine d'années, le modèle unique était la soumission d'un article à une revue. Celle-ci organisait avec son comité éditorial la relecture des manuscrits, effectuait une sélection des articles acceptés après amélioration par les relecteurs, puis s'occupait de la diffusion et de l'archivage des articles. La publication était un objet figé, rarement commenté ou modifié après coup dans la revue, encore plus rarement dénoncé comme frauduleux. L'avènement d'Internet a profondément modifié ce système.

\section{Les pratiques de publication à l'heure du numerique}

On peut aujourd'hui distinguer trois étapes dans la communication des résultats de la recherche : la publication des résultats, la qualification par les pairs et la certification par un éditeur. Ces trois étapes n'en faisaient qu'une dans le système antérieur traditionnel et aboutissaient à ce qu'on nommait " la publication ". Elles sont maintenant largement indépendantes.

Grâce à Internet, il y a toutes sortes de voies pour assurer la publication d'un travail de recherche. On peut, sans contrôle extérieur, le déposer sur un site personnel, sur un blog, sur un site scientifique comme un journal-club. Un minimum de contrôle modérateur permet aussi le dépôt sur une plateforme d'archives ouvertes. Ces dernières assurent la publication d'articles dans leur version de preprint, et aussi de thèses, de communications à des congrès et même de données brutes. Dans le domaine de la physique en France, les plateformes d'archives ouvertes les plus courantes sont ArXiv (site international piloté aux ÉtatsUnis) et HAL (aujourd'hui piloté par le CNRS, l'INRIA et l'université de Lyon).

L'étape suivante, la qualification, concerne l'appréciation du contenu du travail présenté. La méthode la plus courante est le traditionnel peer review ou examen par des collègues considérés compétents dans le domaine concerné, qui tentent d'évaluer l'exactitude des résultats et des conclusions proposées, ainsi que l'originalité, les sources et jusqu'à la forme de l'article. Le peer review se pratique couramment en " simpleaveugle ", c'est-à-dire que les rapporteurs connaissent les auteurs mais pas l'inverse. C'est le cas pour les grandes revues de physique, qu'elles soient traditionnelles ou en open access. Dans d'autres disciplines comme l'informatique, on préfere parfois le "double-aveugle ", supposé éviter l'influence de la notoriété des auteurs sur les rapporteurs, sans que ses avantages supposés fassent l'unanimité. Des usages un peu différents existent dans les grands consortiums de physique et d'astrophysique, qui ont des pratiques de qualification interne.

La dernière étape est la certification de la publication par une communauté éditoriale. Celle-ci la fait paraitre dans le numéro d'une revue scientifique et procède à son archivage dans ses collections. Elle lui attribue en l'enregistrant une indexation permettant sa reconnaissance par les sites comme "web of science" qui comptabilisent les articles parus dans les principales revues. La certification sert aux chercheurs pour l'établissement de leurs listes de publications. 


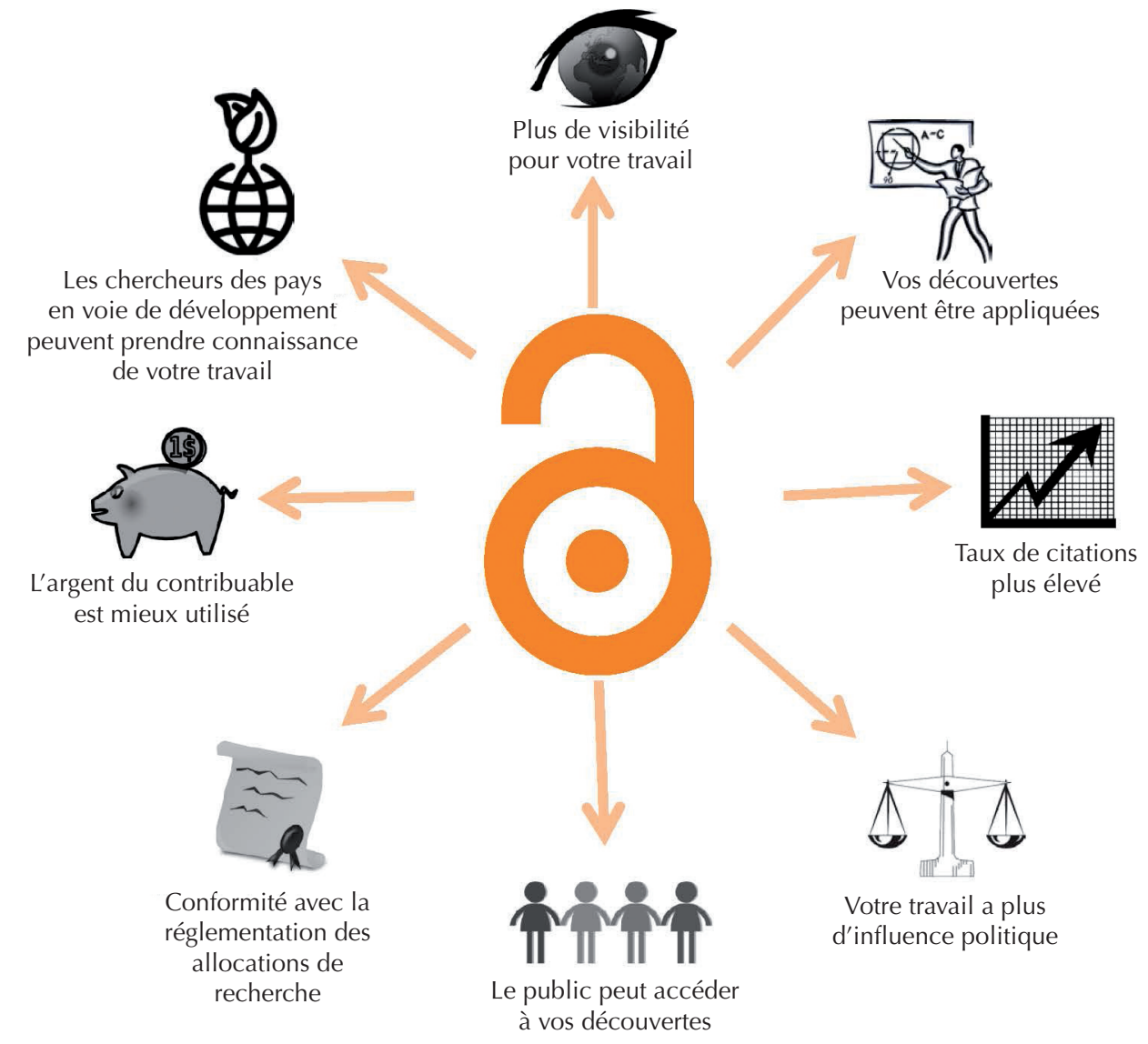

Logo de l'open access et avantages de l'accès ouvert à l'information scientifique et technique.

\section{La qualification par le peer review : un système en crise}

L'étape de qualification par les pairs, assortie de critiques constructives, est en général considérée comme indispensable par la grande majorité des chercheurs. Pourtant le système semble aujourd'hui atteindre ses limites. Un signe en est que des articles publiés par des revues à comité de lecture, y compris les plus prestigieuses, sont contraints à la rétractation en nombre vertigineusement croissant. Le site Retraction Watch qui les recense a été créé en 2010. Les articles sont rétractés en général à la demande de l'éditeur, soit qu'ils contiennent des erreurs ou des résultats trouvés non reproductibles, soit qu'ils soient entachés de malversations telles que des falsifications de données.

Les causes de l'insuffisance présente du peer review sont multiples. La principale tient à l'augmentation considérable du nombre d'articles à évaluer. D'une part, les chercheurs sont poussés par le système de l'évaluation à multiplier les articles pour allonger leur liste de publications. D'autre part, le nombre de revues est en très forte croissance, d'autant plus qu'il se crée de plus en plus de nouvelles revues en open access qui attirent par les coûts très réduits de leurs APC (Article Processing Charges) et engorgent le système. Certaines d'entre elles, dites "prédatrices ", utilisent des comités éditoriaux peu scrupuleux et publient de la science de mauvaise qualité (junk science) $^{(a)}$. Il en résulte une inflation des publications soumises au peer review. Les éditeurs, qui harcèlent les rapporteurs, leur laissent de moins en moins de temps pour l'évaluation des articles soumis (couramment deux semaines, voire une seule), ce qui est incompatible avec un travail de fond. Ainsi, dans un domaine comme les mathématiques, où le rapporteur doit vérifier la validité des démonstrations en les refaisant lui-même, le vrai peer review est devenu vulnérable, voire impraticable. En outre certaines grandes revues prestigieuses, dont Nature, sont susceptibles de fausser la règle du jeu en refusant d'emblée des manuscrits sans les soumettre à l'expertise, ou à l'inverse en forçant l'acceptation d'auteurs de grande notoriété. Enfin, il faut signaler que certaines grandes revues ont été obligées de rétracter un nombre considérable d'articles publiés, parce que les auteurs s'étaient arrangés entre eux pour faire eux-mêmes ou avec leurs amis le peer review de leurs manuscrits ${ }^{(b)}$.

\section{Les réseaux sociaux, nouveaux supports de qualification des publications}

En marge du peer review traditionnel et compte tenu de ses insuffisances, un nombre croissant d'articles font maintenant l'objet de discussions sur les réseaux sociaux, y compris une fois acceptés par des revues. Certaines communautés ont pris l'habitude d'analyser et critiquer les publications scientifiques sur des forums numériques, pour compléter les informations données dans les publications, discuter de la nonreproductibilité de certains travaux, révéler d'éventuelles erreurs, involontaires ou non. Un exemple de ces forums est le site Pubpeer, créé en 2013 par trois chercheurs dont deux travaillent en France dans le 
domaine de la biologie. On est entré dans l'ère dite du PPPR (post-publication-peer review). Ces réseaux scientifiques sur Internet qui se multiplient fournissent des informations, des discussions et dans les cas extrêmes des dénonciations. La distinction entre ces catégories est souvent délicate.

Le cas de Pubpeer est particulièrement intéressant à cet égard. Ses créateurs avaient à l'origine l'unique objectif de permettre des discussions autour des publications et de susciter des échanges $a$ posteriori entre auteurs, rapporteurs et lecteurs. Ils souhaitaient aussi contrebalancer l'importance excessive donnée aux articles dans des revues de prestige pour l'évaluation des chercheurs. Toutefois, quand ils ont choisi d'accepter des commentaires anonymes, ils ont alors enregistré de plus en plus de dénonciations de pratiques douteuses, voire de fraudes, particulièrement dans les domaines touchant aux sciences du vivant. Ils ont alors analysé une situation qu'ils caractérisent par a crisis of trust, symptomatique de la science moderne, victime du système d'évaluation des chercheurs reposant universellement sur le publish or perish.

Notons que si l'anonymat facilité par Internet libère la parole, il peut aussi encourager la malveillance, la mauvaise foi et l'étalage inapproprié de conflits personnels. Les institutions de recherche en France comme le CNRS n'y ont pas recours.

Cependant, les révélations de plus en plus nombreuses de manques à l'intégrité en recherche via les réseaux sociaux concernent un nombre croissant de disciplines. Elles ont conduit en 2016 le ministère de la Recherche à confier une étude sur la question à un comité présidé par Pierre Corvol, professeur au Collège de France, qui prépare des recommandations.

\section{Du bon usage de tous les supports de publication}

Au-delà de leur fonction de contrôle de l'intégrité en recherche, les réseaux sociaux scientifiques, de plus en plus nombreux citons Pubmed Commons, Journal Review, Publons, etc. - agrègent des critiques, des évaluations et des commentaires qui devraient progressivement devenir utiles et bénéfiques. Ils sont ouverts, interactifs, l'information y circule rapidement, ils ne sont pas sous le contrôle des institutions. Notons que les abus des grands éditeurs

En marge du peer review traditionnel

et compte tenu de ses insuffisances, un nombre croissant d'articles font maintenant l'objet de discussions sur les réseaux sociaux [...] Certaines communautés ont pris l'habitude d'analyser et critiquer les publications scientifiques sur des forums numériques, pour compléter les informations données dans les publications, discuter de la non-reproductibilité de certains travaux, révéler d'éventuelles erreurs, involontaires ou non [...] On est entré dans l'ère dite du PPPR (post-publication-peer review).

scientifiques sont de plus en plus souvent dénoncés par les chercheurs, qui doivent payer soit pour voir leurs travaux publiés (dans le modèle open access gold), soit pour pouvoir lire les articles des autres (dans le modèle traditionnel des revues achetées par les bibliothèques), alors que dans tous les cas ils ont conscience de faire l'essentiel du travail : la recherche, les rapports sur les articles, le management éditorial pour les maisons d'édition. Des modèles alternatifs de qualification des publications par Internet, sans éditeurs privés professionnels, commencent à se faire jour. Ils ne sont sans doute pas généralisables, mais fonctionnent déjà dans des communautés assez petites plus ou moins autogérées et pourraient bien tenter de plus en plus de domaines.

Remarquons qu'en physique et en astrophysique, beaucoup d'entre nous déposent déjà leur preprint sur des archives ouvertes dès le jour de leur soumission à des revues. Certains éditeurs tentent encore d'y faire barrage en imposant des embargos. Toutefois la nouvelle loi française pour une République numérique, adoptée le 7 octobre 2016, impose maintenant l'ouverture des résultats obtenus sur fonds publics au bout d'un délai maximum d'un an.

Notons aussi un nouvel avantage du numérique : la trace des chercheurs et de leurs travaux sur Internet permet d'élaborer des graphes de métaconnaissances décrivant les réseaux de chercheurs, avec les liens entre eux et avec leurs thèmes de recherche. Cette nouvelle approche du monde global de la science pourra bientôt être mise à profit par les institutions de recherche, qui auraient intérêt à ne pas en laisser l'usage aux géants de l'Internet.

(a) Voir par exemple : http://openarchive.hypotheses.org/2044

(b) Par exemple Springer a dû rétracter 64 articles dans dix de ses revues en 2014. Voir :

www.nature.com/news/publishing-the-peer-review -scam-1.16400

\section{Pour en savoir plus}

- B. Loveluck, Réseaux, libertés et contrôle. Une généalogie politique d'Internet, Armand Colin (2015)

- https://pubpeer.com/

- www.cnrs.fr/comets/IMG/pdf/mediaaviscometsavril 16-2.pdf

- A. Henri, B. van Tiggelen et M.-A. Leriche, "Réflexions sur la "Voie dorée" des publications scientifiques ", Reflets de la physique 30 (2012) 28-31.

- B. van Tiggelen (tribune), « La SFP et l'INP du CNRS sensibilisent les physiciens à l'utilisation abusive des facteurs d'impact », Reflets de la physique 32 (2013) 46. 